Pathologe 2020 - 41:209-210

https://doi.org/10.1007/s00292-020-00777-w

๑) Springer Medizin Verlag GmbH, ein Teil von Springer Nature 2020

\section{Liebe Leserin, lieber Leser,}

die 104. Jahrestagung der Deutschen Gesellschaft für Pathologie in Berlin hat als thematisches Leitmotiv unser Immunsystem mit besonderem Augenmerk auf dem adaptativen Immunsystem.

Es gibt wohl kein Teilgebiet der $\mathrm{Pa}$ thologie, in dem Immunzellen nicht eine wesentliche Dimension der Organ- und der Systemerkrankungen darstellen. Bei vielen Organkrankheiten nimmt man das Immunsystem ganz global wahr, subsumiert im Konzept der chronischen Entzündung und deren unterschiedlichen histologischen Erscheinungsformen. Dann tritt es uns auch als Organsystem in Form strukturierter lymphatischer Gewebe entgegen. Dabei ist ein Immunzellinfiltrat in einem nichtlymphatischen Gewebe grundsätzlich eine zielgerichtete konzertierte Aktion, teleologisch gesehen ein Angriff auf und/oder Abwehr von etwas, das das Immunsystem als schädlich identifiziert hat. Die ständige Aktivität in den lymphatischen Geweben indessen dient der Aufrechterhaltung der Selbsttoleranz und der Remobilisierung gegen ehemals erfolgreich bekämpfte Noxen und der Erkennung neuer Noxen sowie der Generierung einer adäquaten Antwort darauf. Unser Immunsystem hält ein Leben lang DNA-Viren wie Zytomegalieviren (CMV), Herpesviren und Epstein-Barr-Viren (EBV), die, einmal erworben, nicht komplett eliminierbar sind, in Schach, ohne Aufhebens davon zu machen. Ebenso wird wohl sehr viel anderes Infektiologisches in aller Diskretion, sprich ohne Klinik, vom Immunsystem im Sinne einer „stillen Feiung“" (Pfaundler) erledigt und trägt so in einem durchaus aversiven mikro-

\title{
Peter Möller
}

Institut für Pathologie, Universitätsklinikum UIm, Ulm, Deutschland

\section{Unser Immunsystem - der Staat im Staate}

biologischen Umfeld, das uns umgibt, wesentlich zum Gesundsein bei.

Diesen lebenswichtigen und -erhaltenden Effekten stehen Immundefizienz und Autoimmunität als krankheitsverursachendes Versagen dieses Schutzsystems entgegen. Bei Neoplasien - immuntheoretisch argumentiert Erscheinungen in der Interphase zwischen Selbst und Nicht-mehr-Selbst - ist es entweder zu tolerant oder wird von der Neoplasie aktiv paralysiert. Und nicht zuletzt kann das Immunsystem selbst erkranken, durch lymphotrope Viren wie EBV und HIV und durch eine maligne Transformation in Immunzellen, woraus dann die komplexe Welt der Lymphome resultiert.

Unserem Immunsystem ist diese Tagung gewidmet. Sie beginnt im Themenblock „Immunpathologie I“ mit Beiträgen zu angeborenen Immundefekten. Dazu gibt es einen Beitrag in diesem Themenheft von den Autoren Posovszky und Barth, die eine Ulmer Kohorte von MHC-Klasse-II-defekten Kindern mit intestinaler Dystrophie vor und nach allogener Knochenmarkstransplantation untersucht haben. Weiter fällt der Fokus auf den Makrophagen als die die Immunreaktionen und akute Entzündung orchestrierende Zelle, welche hier in ihrer funktionellen Diversität vorgestellt wird.

Das Immunsystem des Fettgewebes ist erst ansatzweise verstanden. Frau Fischer-Posovszkyist eine internationalanerkannte Forscherin auf diesem Gebiet und hat ihren Vortrag auf dieser Tagung als Beitrag für dieses Themenheft verschriftlicht.

Der Themenblock „Immunpathologie II“ widmet sich zunächst dem humanen Mikrobiom, gefolgt von Beiträgen über Immunproteomics und persistie- rende T-Zell-Konotypen bei refraktärer Zöliakie sowie einem Übersichtsreferat von Frau Beck zu Autoimmunenteropathien, welches in diesem Themenheft $\mathrm{zu}$ lesen ist. Thema der Thymuspathologie sind mit der lymphofollikulären Thymushyperplasie assoziierte Lymphome. Das Komplementsystem ist in letzter Zeit besonders in der Nephropathologie interessant geworden, was in diesem Themenheft von Frau Amann beschrieben wird.

Der Themenblock „Immunpathologie III" beginnt mit Beiträgen zur Rolle des inflammatorischen Mikromilieus in soliden Tumoren, über die Herr Denkert in diesem Themenheft ein Update gibt. Weitere Beiträge fokussieren und analysieren diese Immunreaktionen im Ösophagus-, im Magen-, im Kolonkarzinom und im pulmonalen Adenokarzinom.

Die Themenblöcke über die „Immunonkologie“ beginnen mit einer State-ofthe-art-Lecture über Immunrezeptorsignale in Neoplasien. Danach folgen weitere Beiträge $\mathrm{zu}$ intratumoralen Immunreaktionen. Dazu gibt es ein Übersichtsreferat über das Mikroenvironment des Hodgkin-Lymphoms von Frau Mottok, das als letzter themenbezogener Beitrag in diesem Heft erscheint. Aus dem Lymphomgebiet gibt es Beiträge über Zellmigration im klassischen Hodgkin-Lymphom und dem großzellig-anaplastischen Lymphom sowie Beiträge zur Th17-Achse im follikulären Lymphom. Ein Meeting Highlight ist Herr Viardot, der über Erfahrungen der Lymphomonkologen mit CAR-T-ZellTherapien sprechen wird. Weitere Themen wie invariante natürliche Killer TZellen (iNKT), Immune-Escape-Mechanismen und metabolische Einflüsse auf die Anti-Tumor-Immunität sowie PD-1/ 
PD-L1 und deren prädiktive Bedeutung im cholangiozellulären Karzinom, im Lungenkarzinom, im kutanen T-ZellLymphom und im Harnblasenkarzinom werden ebenso zur "Immunonkologie“ beitragen wie Referate zur biomarkerbasierten Prädiktion des therapeutischen Ansprechens beim malignen Melanom.

Weitere Meeting Highlights sind Gastredner aus der deutschen Immunologie: Frau Romagnani referiert über „Tissue residency and ILCs" und Herr Rodewald über „Deconvolution of hematopoiesis by fate mapping, barcoding, and single cell transcriptomics".

Jeder, der Pathologie diagnostisch und wissenschaftlich betreibt, hat in seiner Arbeit mit dem Immunsystem zu tun. Die wissenschaftliche Pathologie hat sich in den letzten 20 Jahren erstaunlich entwickelt und dabei auch leider sehr diversifiziert, wofür die vielen Arbeitsgemeinschaften der DGP beredter Ausdruck sind. Die 104. Jahrestagung will mit dem wissenschaftlichen Hauptprogramm alle Subdisziplinen zusammenbringen und den Teilnehmerinnen und Teilnehmern eine Übersicht über weite Teile unseres Faches vermitteln.

Ich hoffe, dass das vorliegende Heft von Der Pathologe schöne Erinnerungen an die 104. Jahrestagung der DGP erweckt.

Mit freundlichen kollegialen Grüßen,

Ihr

P. Möller

\section{Korrespondenzadresse}

Prof. Dr. Peter Möller

Institut für Pathologie, Universitätsklinikum Ulm

89070 Ulm, Deutschland

Peter.Moeller@uniklinik-ulm.de

Interessenkonflikt. P. Möller gibt an, dass kein Interessenkonflikt besteht.

\section{Weltweit erstes Modell für das frühe Stadium der Arthrose}

Arthrose ist ein großes gesellschaftliches Problem: Alleine in Deutschland leiden rund fünf Millionen Menschen an Arthrose. Die Frühphase der Krankheit ist dabei noch relativ unerforscht. Dem Arthroseforscher Henning Madry ist es nun erstmals gelungen, die Frühphase der Krankheit im Kniegelenk genauer zu beobachten. Das Modell des Krankheitsverlaufs hat er in „Science Translational Medicine“ veröffentlicht.

Ca. $83 \%$ der Betroffenen leidet an einer Kniegelenksarthrose. Schmerzen und eingeschränkte Bewegungsfreiheit sind die langfristigen Folgen. Dabei ist vielen häufig gar nicht bewusst, dass sie an Arthrose leiden. „Das ist ein schleichender Prozess", sagt Henning Madry, Professor für Experimentelle Orthopädie und Arthroseforschung an der Saar-Uni und Direktor des Zentrums für Experimentelle Orthopädie am Universitätsklinikum des Saarlandes. „Irgendwann tut ihnen das Knie weh, ohne dass sie zuvor Schmerzen hatten. Dabei haben viele schon jahrelang Arthrose, ohne es zu merken", erläutert der Wissenschaftler den Krankheitsverlauf. Kommen die Patienten dann zu ihm in die Klinik, ist die Krankheit oft schon in einem fortgeschrittenen Stadium.

Deshalb suchen Arthroseforscher und Orthopäden wie Madry nach den Ursachen der Krankheit. Mit diesem tieferen Verständnis kann es dann gelingen, die Krankheit besser vorherzusagen und so einem Arthrosepatienten womöglich einen langen Leidensweg und größere Operationen zu ersparen.

Madry und seinem Team ist es nun gelungen, die Kniegelenksarthrose im Frühstadium besser zu verstehen. Dabei hat er sich auf einen bereits bekannten Umstand gestützt: Arthrose entsteht fast immer, wenn der Meniskus geschädigt ist. „Was man allerdings nicht weiß: Wie genau verläuft der Weg vom Meniskusschaden zu Schäden am Gelenkknorpel und damit zur Arthrose?", fragt Professor Madry. „Aus klinischen Studien wissen wir, dass der Verlust des Gelenkknorpels nicht gleichmäßig, sondern hier und da unterschiedlich verläuft."

Genau auf diese Frage konnte er nun experimentell eine Antwort finden und ein Modell entwickeln, das Forscher künftig heranziehen können, wenn sie den Krankheitsverlauf vorhersagen möchten. Dazu sind Madry und seine Mitarbeiterinnen und Mitarbeiter in drei Schritten vorgegangen. „Zuerst haben wir uns bei Patienten angeschaut, wo genau der Meniskusschaden ist. Dann haben wir den Knorpelschaden genau lokalisiert", erläutert Madry den ersten Schritt. In einem zweiten Schritt haben die experimentellen Orthopäden die Kniegelenke von Schafen mit Arthrose untersucht, zuerst in einem frühen Stadium und dann in einem späten Stadium. „Hier konnten wir sehr genau im Knorpel und im Knochen nachschauen, was wann passiert im Verlauf der Krankheit", so Madry über diesen Schritt der Forschungsarbeit. Sie fanden unter anderem heraus, dass Knorpel- und Knochenabbau zuerst an der Stelle der Verletzung auftraten, bevor sie sich in der gesamten Kniegelenksregion ausbreiteten.

In einem dritten Schritt haben die Forscher wiederum Patienten mit schwerer Arthrose untersucht und mit der späten Verlaufsform der Schafs-Arthrose verglichen. „Wir haben dabei festgestellt, dass die menschlichen Arthrosen und die Arthrosen im Schafsmodell nach Meniskusschaden ganz ähnlich verlaufen", erläutert Madry. Mit den so erstellten hochauflösenden topographischen Karten des Krankheitsverlaufs kann genau die Pathologie der menschlichen Arthrose widergespiegelt werden. Dies ist der Beweis dafür, dass die beobachteten Verläufe bei den Schafen als Modell für die Entwicklung der Krankheit auch beim Menschen dienen können.

Diese Erkenntnisse zum räumlichen und zeitlichen Arthroseverlauf sind bisher weltweit einzigartig. Mit ihrer Studie haben Madry und sein Team Arthroseforschern weltweit ein Werkzeug an die Hand gegeben, um weiter an den Ursachen der Krankheit zu forschen und für künftige Patienten bessere Therapie- und auch Präventionsmaßnahmen zu entwickeln.

Originalpublikation:

T. Oláh, J.Reinhard, L. Gao et al. (2019), Topographic modeling of early human osteoarthritis in sheep. ScienceTranslational Medicine, DOI: 10.1126/scitransImed.aax6775. 